\title{
The Influence of Humanistic Care on the Mental Health and Behavior of Family Members of Pediatric Patients
}

\author{
Lijun Chang \\ Children's Hospital of Soochow University, Suzhou 215000, Jiangsu, China \\ Email: 244595192@qq.com
}

\begin{abstract}
The purpose of this paper is to observe and analyze the impact of humanistic care on the mental health and behavior of family members of pediatric patients. The methods used are as follows. A total of 188 hospitalized children (188 cases of family members of children) admitted to the pediatrics department of this hospital were selected. In accordance with random number table method, they were divided into the control group (using routine nursing methods) and the experimental group (using humanistic care nursing methods on the basis of the control group). There were 94 cases in each group. Statistics was used to analyze the total satisfaction rate, the incidence of nurse-patient disputes, the average hospitalization time of the patients and the anxiety scores of the family members of the children before and after nursing in the two groups. The anxiety scores of the family members of the two groups of children before nursing were not statistically significant. After nursing, the anxiety scores of the family members of the experimental group were significantly lower than that of the control group. The total satisfaction rate of the family members of the children in the experimental group was significantly higher than that in the control group. And the incidence of nurse-patient disputes in the experimental group was significantly lower than that in the control group. The average hospitalization time of the children in the experimental group was significantly shorter than that in the control group. The conclusion is that humanistic care has a significant effect on the mental health and behavior of the family members of pediatric patients.
\end{abstract}

Keywords: humanistic care, family members of pediatric patients, mental health and behavior, influence

\section{Introduction}

Paediatric nursing work is significantly different from nursing work in other departments. Pediatric patients have poor self-expression ability because they are young, so they need to express themselves through family members. Investigations and studies have shown that the common mental states of family members of pediatric patients are as follows: first, anxiety; second, suspicion; third, tolerance; fourth, fear, etc. ${ }^{[1]}$ The psychological state of the children's family members has a direct impact on the children's nursing and treatment, and it is particularly important to carry out humanistic care for the family members of pediatric patients.

\section{Materials and methods}

\subsection{General information}

A total of 188 hospitalized children (188 cases of family members of children) admitted to the hospital's pediatric department (from April 2018 to April 2019) were selected. Inclusion criteria: (1) the family members of the pediatric patients are the fathers or mothers of the children; (2) the family members of the children who participate are all informed. Exclusion criteria: (1) those with mental retardation; (2) those who can't express themselves in words. In the experimental group, there were 46 male children and 48 female children, with an average age of (4.5 \pm 3.3 ). Types of disease: 27 cases of upper respiratory tract infection, 36 cases of asthma, 20 cases of pneumonia and 11 cases of other diseases. In the experimental group, there were 52 females and 42 males, with an average age of (29.6 \pm 3.8$)$. In the control group, there were 49 male children and 45 female children, with an average age of (4.9 \pm 2.7$)$. Disease types: 30 cases of upper respiratory tract infection, 33 cases of asthma, 22 cases of pneumonia, and 9 cases of other diseases. In the experimental group, there were 54 females and 40 males, with an average age of $(30.3 \pm 3.5)$.

\subsection{Methods}

Routine nursing methods were used in the control group, and the main nursing contents were as follows. First, basic disease observation; second, diet guidance; third, routine education and so on. The nursing method of humanistic care was applied in the experimental group on the basis of the control group, and the main nursing contents were as follows. First, cultivate nurses' concept of humanistic care. Organize pediatric nurses to 
systematically study the knowledge of humanistic care. Make them fully grasp the relevant theories and important values, grasp the psychological characteristics of children's families, accurately communicate with children's families, and fully respect the children's values and privacy. Second, create a good ward environment. The ward was arranged in a family style so that the children could feel safe and comfortable. Maintain proper light in the ward, post animated posters on the wall, or display toys, etc. When the children's condition permitted, let them watch cartoons, and explain the causes of the disease and related nursing knowledge to the family members of the children through pictures or lectures, etc. so as to increase the family members' confidence in treatment. Third, strengthen the psychological nursing of the family members of the children. Nurses needed to warmly receive the family members of children and make them feel cordial from the aspects of action and language, so as to create a good nurse-patient relationship. Primary nurses should take the initiative to introduce the main points of nursing and related precautions to the family members, so as to reduce the excessive tension caused by the family members' ignorance of the disease. Symposiums were also held regularly to answer questions from the children's family members in time, so that the family members could actively cooperate with medical staff on relevant treatment. Some family members had negative psychological emotions due to high medical costs. Nurses should take the initiative to explain the items and sources of medical costs to them, and seek support from the social system in order to alleviate their pressure. Nurses should also organize exchanges of psychological care with family members of the same disease to ease their pressure Fourth, strengthen health education. Nurses could make full use of nursing operation time to carry out targeted health education for the family members of the children, deepen their awareness of the disease and related treatment methods, and eliminate the fear of the family members of the children. In addition, establish a harmonious nurse-patient relationship. The medical staff maintained a serious and responsible attitude and gained the trust of the children's families. Medical staff needed to seize the opportunity to communicate with the family members of the children in the course of work, so as to gain their trust. By explaining the knowledge of life nursing to the patients' family members, nursing staff continued to help them build up confidence and then alleviate their psychological pressure.

\subsection{Observation indicators}

The total satisfaction rate of the two groups family members of pediatric patients, the incidence of nurse-patient disputes, the average hospitalization time of the children and the anxiety scores of the family members of the children before and after nursing are analyzed.

\subsection{Statistical methods}

Data analysis is performed by using SPSS 21.0 statistical software.

\section{Results}

\subsection{Comparison of anxiety scores of the two groups of family members of children before and after nursing}

The comparison of the anxiety scores of the two groups of family members of children before nursing is not statistically significant $(\mathrm{P}>0.05)$. After nursing, the anxiety scores of the family members of the children in the experimental group is significantly lower than those in the control group $(\mathrm{P}<0.05)$. See Table 1 .

Table 1 Comparison of anxiety scores of the two groups of family members of children before and after nursing (points)

\begin{tabular}{ccc}
\hline Groups & Before nursing & After nursing \\
\hline The experimental group $(\mathrm{n}=94)$ & $41.4 \pm 2.4$ & $20.3 \pm 1.1$ \\
The control group $\quad(\mathrm{n}=94)$ & $42.0 \pm 2.0$ & $29.6 \pm 1.8$ \\
$\mathrm{t}$ & 12.4556 & 25.5638 \\
$\mathrm{P}$ & 0.0635 & 0.0214 \\
\hline
\end{tabular}

\subsection{Comparison of indicators such as the total satisfaction rate of the family members of the two groups, the incidence of nurse-patient disputes and the average hospitalization time of the children}

The total satisfaction rate of the family members of the children in the experimental group is significantly higher than that in the control group $(\mathrm{P}<0.05)$. The incidence of nurse-patient disputes in the experimental group is significantly lower than that in the control group $(\mathrm{P}<0.05)$. The average hospitalization time of the children in the experimental group is 
significantly shorter than that in the control group $(\mathrm{P}<0.05)$. See Table 2 .

\begin{tabular}{|c|c|c|c|}
\hline Groups & The total satisfaction rate $(\mathrm{n} / \%)$ & $\begin{array}{l}\text { The incidence of nursing } \\
\text { disputes }(\mathrm{n} / \%)\end{array}$ & $\begin{array}{l}\text { The average hospitalization } \\
\text { time of the children (d) }\end{array}$ \\
\hline The experimental group $(\mathrm{n}=94)$ & $92(97.9)$ & $2(2.1)$ & $7.5 \pm 2.4$ \\
\hline The control group $(\mathrm{n}=94)$ & $80(85.1)$ & $14(14.9)$ & $12.6 \pm 2.8$ \\
\hline $\mathrm{t}$ & 12.5457 & 20.1269 & 28.1233 \\
\hline $\mathrm{P}$ & 0.0256 & 0.0102 & 0.0000 \\
\hline
\end{tabular}

\section{Discussion}

Pediatrics is one of the important departments in the hospital. Because the children's ability to express is not strong and lack of self-care ability, the treatment and nursing process requires the cooperation of the children's family members ${ }^{[2]}$. With the change of the nursing model, people pay more and more attention to the psychological status of the children's family members. Family members are the main supporters of the children, and the existence of negative psychological emotions of the family members will affect the rehabilitation status of the children ${ }^{[3]}$.

In the process of nursing children, pediatric nurses need to face not only the children who can't express themselves, but also the family members who have too high expectations for nursing and do not master comprehensive medical knowledge. Therefore, the nursing difficulty of pediatric nurses has increased significantly ${ }^{[4]}$. Only when the pediatric nurses master skilled professional operation technology and has rich nursing knowledge can the nursing work be carried out better. In the process of implementation, humanistic care can continuously improve the nursing level of pediatric nurses, and can effectively relieve the negative psychological emotions of the children's family members. It can ensure that children receive the relevant treatment and care smoothly and helps maintain a good nurse-patient relationship and doctor-patient relationship. The implementation of the humanistic care nursing model can effectively relieve the tension and anxiety of the children's family members, strengthen communication between nurses and patients, and increase mutual understanding. Moreover, it can increase the nursing effectiveness of the children's family members, and further improve their total nursing satisfaction ${ }^{[5-6]}$.

The results of this study have shown that the anxiety scores of the two groups of the family members of children before nursing are not statistically significant ( $>>0.05)$. After nursing, the anxiety scores of the family members of children in the experimental group is significantly lower than that in the control group $(\mathrm{P}<0.05)$; the total satisfaction rate of the family members of the children in the experimental group was significantly higher than that of the control group $(\mathrm{P}<0.05)$; Compared with the control group $(\mathrm{P}<0.05)$; the average hospital stay of the experimental group was significantly shorter than that of the control group $(\mathrm{P}<0.05)$. The above research results show that humanistic care has a significant effect on the mental health and behavior of the family members of pediatric patients. Humanistic care can fully take the patients as the center. By creating a warm ward environment, strengthening health education and psychological intervention, etc., humanistic care can affect the cognition and behavior of the family members of the children, reduce the negative impact of negative psychological emotions on the children, and finally eliminate the sense of tension and anxiety of the family members of the children ${ }^{[7-8]}$. Humanistic care can also improve the awareness of children's family members and nurses, and help maintain a good nurse-patient relationship. In addition, humanistic care is conducive to strengthening communication between nurses and patients, reducing contradictions, enhancing the confidence of family members of children in the treatment effect, continuously improving the overall satisfaction rate of nursing, and shortening the hospitalization time of children.

\section{References}

[1] Tan Weiqing, Chen Qiao, Zhen Qinglian. Application of health education of children 's family members in closed management of neonatal wards. Nursing Practice and Research. 2019; 16 (14): 125-127.

[2] Wang Wenchao, Hu Jing, Gu Ying, et al. Self-assessment of hospice and cognition level of medical staff in pediatric intensive care unit. Journal of Qilu Nursing. 2019; 25 (6): 49-52

[3] Sun $\mathrm{Xu}$, Han $\mathrm{Lu}$, Gao Wenwen. Strengthen risk management to reduce the incidence of pediatric medical disputes. Contemporary Medicine. 2020; 26 (1): 119-120. 
[4] Chen Liyu, Xu Zijun. Survey on anxiety status of sick children's family members in Ordinary Pediatrics of Guangdong Provincial Hospital of Traditional Chinese Medicine and its influencing factors. Occupation and Health. 2017; 33 (6): 783-786.

[5] Wang Tong. Application of competency-oriented management in the management of pediatric nurses. International Journal of Nursing. 2018; 37 (19): 2705-2708.

[6] Liu Lan. Explore the application effect of risk prevention nursing in pediatric nursing management. Journal of Clinic Nursing's Practicality. 2018; 3 (40): 160-161.

[7] Pan Chengjia. Analysis of the application effect of high-quality nursing services in pediatric nursing work. Journal of Clinic Nursing's Practicality. 2018; 3 (19): 128.

[8] Lin Qiulian, Shi Wenxiang, Zhang Shuqin. Application of clinical path nursing combined with psychological nursing in children undergoing laparoscopic repair of indirectinguinal hernia. Journal of Qilu Nursing. 2019; 25 (12): $37-39$. 